\title{
Chromosomal Concordance between the Baby Produced by Preimplantation Genetic Testing Embryo and the Trophectoderm Biopsy: a Retrospective Study
}

\section{Zhongyuan Yao}

Center for Medical Genetics \&amp; Hunan Key Laboratory of Medical Genetics, School of Life Sciences, Central South University

Xiaoxia Wang ( $\triangle$ xiaoiawang@163.com)

Department of Reproductive Medicine, Xiangya Hospital, Central South University https://orcid.org/0000-0002-3848-2821

Jun Zeng

Department of Reproductive Medicine, Xiangya Hospital, Central South University Jing Zhao

Department of Reproductive Medicine, Xiangya Hospital, Central South University

\section{Qiuping Xia}

Department of Reproductive Medicine, Xiangya Hospital, Central South Univeristy

\section{Lei Zhang}

Department of Reproductive Medicine, Xiangya Hospital, Central South University

\section{Lingqian Wu}

State Key Laboratory of Medical Genetics

\section{Yanping Li}

Department of Reproductive Medicine, Xiangya Hospital, Central South University https://orcid.org/0000-0002-4193-7321

\section{Research Article}

Keywords: Preimplantation genetic testing, trophectoderm biopsy, next generation sequencing, mosaicism, aneuploidy, self-correction

Posted Date: March 30th, 2021

DOl: https://doi.org/10.21203/rs.3.rs-364582/v1

License: (c) (i) This work is licensed under a Creative Commons Attribution 4.0 International License. Read Full License 



\section{Title Page}

Title: Chromosomal Concordance between the Baby Produced by Preimplantation Genetic Testing Embryo and the Trophectoderm Biopsy: a Retrospective Study

Author information:

Zhongyuan Yao, Ph.Da,b,c, Xiaoxia Wang, M.D.b, Jun Zeng, M.D.b, Jing Zhao, M.D.b,c, Qiuping Xiab,c, Ph.D, Lei Zhang, M.M.b, Lingqian Wu*a, Ph.D, Yanping Li*b,c, M.D.

a Center for Medical Genetics \& Hunan Key Laboratory of Medical Genetics, School of Life Sciences, Central South University, Changsha, Hunan, 410078, China

b Department of Reproductive Medicine, Xiangya Hospital, Central South University, Changsha, Hunan,410078, China.

${ }^{\mathrm{C}}$ Clinical Research Center for Women's Reproductive Health in Hunan Province, Hunan,410087, China Zhongyuan Yao and Xiaoxia Wang contributed equally in this study.

\section{Corresponding author:}

Yanping Li, Reproductive Medicine Center, Xiangya Hospital, Central South University, Changsha, Hunan,410000, People's Republic of China. TEL: +86 13607317798. E-mail: liyanp@csu.edu.cn Lingqian Wu, Center for Medical Genetics \& Hunan Key Laboratory of Medical Genetics, School of Life Sciences, Central South University, Changsha, Hunan, 410078, China. E-mail: wulingqian@sklmg.edu.cn 


\section{Chromosomal Concordance between the Baby Produced by Preimplantation Genetic Testing}

Embryo and the Trophectoderm Biopsy: a Retrospective Study

\section{Abstract}

Background: Chromosomal mosaicism and aneuploidies are routine phenomena throughout human preand post-implantation development. The benefit of implanting mosaicism or aneuploidies is still controversial. The purposes of the study are to investigate the developmental potential of embryos with chromosomally segmental or mosaic abnormalities, and whether precise Next Generation Sequencing (NGS) resolution would reduce the development of an abnormal embryo in preimplantation genetic testing (PGT) cycles.

Methods: The peripheral blood of 17 PGT babies were collected for single nucleotide polymorphism (SNP) array and were compared with trophectoderm (TE) biopsy results at different NGS resolutions.

Results: $76.5 \%$ (13/17) of babies' peripheral blood chromosome analysis was consistent with 10Mb TE biopsies and 58.8\% (10/17) of babies' analysis was consistent with 4Mb TE biopsies. 2 babies who had euploid TE showed abnormal peripheral blood chromosome analysis. 17.6\% (3/17) embryos with aberrant TE biopsies produced healthy babies. Although the sensitivity of $10 \mathrm{Mb}$ was lower than $4 \mathrm{Mb}$ ( $25 \%$ vs. $50 \%$ ), the specificity (100\% vs. $76.9 \%)$, PPV (100\% vs. $40 \%)$ and diagnostic accuracy $(82.4 \%$ vs. $70.6 \%)$ of $10 \mathrm{Mb}$ showed better results than $4 \mathrm{Mb}$.

Conclusion(s): The chromosomal results between peripheral blood samples and TE biopsies of born babies are not completely congruent. Aneuploid and mosaic embryos have potential to produce healthy babies, whereas normal embryos also have chance to produce babies with chromosomal abnormalities. In spite of low sensitivity of both resolutions, $10 \mathrm{Mb}$ has higher specificity, PPV and diagnostic accuracy than $4 \mathrm{Mb}$. It is suggested that TE biopsy be analyzed in both $10 \mathrm{Mb}$ and $4 \mathrm{Mb}$ resolutions to uncover severely adverse chromosomal aberrations but use $10 \mathrm{Mb}$ resolution to guide transfer.

Trial registration: The study was retrospectively registered in the Chinese Clinical Trial Registry (ChiCTR2100042522).

Keywords: Preimplantation genetic testing; trophectoderm biopsy; next generation sequencing; mosaicism; aneuploidy; self-correction

\section{Background}

Since the first preimplantation genetic testing (PGT) baby was born in 1989 (1), more patients with chromosomal diseases, monogenetic diseases, or even recurrent miscarriages have opportunities to have their own babies. However, not all patients will obtain normal embryos through PGT cycles. Sometimes, after comprehensive considerations and adequately counseling with patients, embryos with partial genetic problems have been transferred into uterus. Strange and interesting phenomena during the followup that those embryos have potential to produce healthy children were reported (2-4). Of note, TE biopsies were used for PGT in those studies for not damaging embryos, which were not direct to reflect the inner cell mass (ICM). These discoveries lead us to think about a question: is PGT method using TE biopsy accurate enough to predict the developmental potential of an embryo? To date, there is no consensus whether it is beneficial to implant embryos with chromosomal abnormalities in the clinic. Furthermore, most studies focused on the chromosomal pattern of the babies born by implanting mosaic or aneuploid embryos, not euploid embryos $(2,4)$. There were few studies on chromosomal copy number variations (CNVs) of born babies after implanting euploid embryos. Thus, it is also worth discussing the CNV concordance between normal PGT embryos and born babies. 
PGT techniques have evolved quickly, from fluorescence in situ hybridization (FISH), to array comparative genomic hybridization $(\mathrm{aCGH})$, single nucleotide polymorphism (SNP) array, and more recently, next generation sequencing (NGS)(5). Among them, NGS is an emerging technology that provides unprecedented high-throughput, highly parallel, and base-pair resolution data for genetic analysis (6). Obviously, it shows better application than other technologies with superior sensitivity and higher precision for chromosomal analysis, and now it is possible to detect chromosomal mosaicism at levels between $20 \%-80 \%(4,7-10)$. However, the benefits of such a high-resolution technique that detects more embryos with chromosomal aberrations which perhaps result in the discard of some potentially viable blastocytes remains unclear.

We hypothesized there possibly existed allegedly euploid embryos in rough NGS resolution which were implanted and gave birth to babies in our department. Moreover, we hypothesized that finer NGS resolution would reduce the chance of some mosaic and aneuploid embryos to develop healthy babies. Thus, the present retrospective study presented and estimated the chromosomal concordance between peripheral blood samples of born PGT babies and NGS resolutions in $4 \mathrm{Mb}$ and $10 \mathrm{Mb}$ to explore the accuracy of TE biopsy and whether precise NGS resolution should be applied into the clinical practice.

\section{Methods}

\section{Study Design and Ethical Permission}

The study was approved by the Institutional Review Board, Department of Reproductive Center, Xiangya Hospital, Central South University (No.202006) and registered in the Chinese Clinical Trial Registry (ChiCTR2100042522). In our department, 10Mb NGS resolution was conducted to analyze TE biopsy for PGT before 2019, but now the NGS resolution could reach $4 \mathrm{Mb}$ or even more precise. Not regarding normal embryos or abnormal embryos implanted, a comprehensive search was conducted in the database of from January 2017 to December 2018. The inclusion criteria: (1) couples were treated by PGT; (2) couples gave birth to a baby. All couples that met the criteria were called to ask about their wills to participate in the study. After receiving their approval and written informed consent forms, the preserved DNA amplified samples of their implanted embryos were sent to retested by NGS at $4 \mathrm{Mb}$ resolution and their children's peripherical blood samples were collected for SNP array analysis to confirm CNVs.

\section{Embryo Culture and Biopsy}

For patients required with PGT, controlled ovarian stimulation was performed according to conventional protocols. Transvaginal oocyte retrieval was then scheduled for 36 hours later after hCG was administrated to induce final oocyte maturation. Embryos were all fertilized by intracytoplasmic sperm injection (ICSI) in order to eliminate the risk of contamination from sperm DNA, and then cultured in cleavage embryo culture medium (COOK, K-SICM-50) and subsequently blastocyte culture medium (COOK, K-SIBM-50). Until Day 5 or Day 6 of development, blastocytes were scored based on the Gardner system(11) and then a single laser pulse using Saturn ${ }^{\text {TM }}$ Lasers Systems (CooperSurgical Inc., USA) was carefully applied in assistance to blastocoel hatching, providing a safe distance from the ICM. 5-8 cells would be sucked for TE biopsy was performed in the expanded blastocytes after 5-6 hours, and all biopsied embryos were then vitrified.

\section{Frozen embryo transfer}

A selected embryo was transferred in the next frozen cycle after careful considerations by reproductive 
clinicians and embryologists until the NGS results came out. Blastocyst vitrification and warming methods were described as before(12). During the frozen embryo transfer (FET) cycle, hormone replacement therapy (HRT) or natural cycle (NC) were chosen for personalized treatment depending on patients. In HRT-FET cycle, estrogen pills were used to prepare endometrium for approximately 12 days. Then progesterone pills were administered for endometrial transformation. In NC-FET, clinicians monitored development of the dominant follicle until it was discharged and the progesterone pills were applied for corpus luteum support.

\section{Sample Processing and Testing}

All trophectoderm (TE) samples used in the study had been processed by multiple annealing and loopingbased amplification cycles. After approval, the saved TE amplified samples belonging to participants were sent for NGS to be retested at resolutions of $4 \mathrm{Mb}$. Additionally, the peripheral blood samples were collected from participants for SNP array. CNVs was detected in both kinds of samples. And all testing was conducted by Yikon Genomics according to the methods as they previously described(13-15). 30\%$70 \%$ abnormal cells were classified as mosaicism. Otherwise, euploidy or aneuploidy was reported. For $10 \mathrm{Mb}$ resolution, $1 \mathrm{M}$ reads were obtained from one reaction in high-throughput sequencing, the sequencing length was approximately $50 \mathrm{bp}$, and the depth was around $0.025 \mathrm{X}$. As to $4 \mathrm{Mb}$ resolution, $1.5 \mathrm{M}$ reads could be obtained in one reaction, the sequencing length was around $50 \mathrm{bp}$, and the depth reached $0.025 \mathrm{X}-0.040 \mathrm{X}$.

\section{NGS Protocol Validation}

Before initiation of our study, the validation of NGS protocol was performed for accuracy. 3 Preimplantation Genetic Testing - Aneuploidy Kits (Semiconductor sequencing) (Yikon, H170601, H170602, H170603) were used in 36 positive reference materials (Yikon, H170301) for library construction. Then libraries were sequenced with a DA8600 sequencer. Every sample was tested 3 times. 4 negative reference materials (Yikon Genomics, H170301) and 4 mosaic reference materials (Yikon Genomics, H170301) were conducted as positive reference materials. The results were compared with the preset reference sample indicators. For quality control, control samples (positive control: 21-Trisomy; negative control: $46, \mathrm{XN}$; blank control) were required to be detected when all reference samples were detected. The total library construction failure rate should not be more than $3 \%$, the valid data should not be lower than $1 \mathrm{Mb}$ and the genomic coverage should be not lower than $4 \%$. All tested PGT-A kits met the accuracy standards and could be used for clinical sample sequencing (data were provided by Yikon Genomics and not shown here).

\section{Statistical Analysis}

GraphPad Prism 8 was applied for statistical analysis and graph preparation. Categorical variables were assessed by Chi-square test or Fisher exact test. Sensitivity, specificity, diagnostic accuracy, positive predictive value (PPV) and negative predictive value (NPV) for $10 \mathrm{Mb}$ and $4 \mathrm{Mb}$ NGS resolutions were calculated as follows: (1) Sensitivity $=\frac{\text { True positives }}{\text { True positives }+ \text { False negatives }} \times 100$, (2) Specificity $=$

\footnotetext{
$\frac{\text { True negatives }}{\text { True negatives }+ \text { Fakse positives }} \times 100 \quad$ (3) $\quad$ Diagnostic accuracy $=$ $\frac{\text { True positives }+ \text { True negatives }}{\text { True positives }+ \text { False positives }+ \text { False negatives }+ \text { True negatives }} \times 100$, (4)Positive predictive value $=$ $\frac{\text { True positives }+ \text { True negatives }}{\text { True positives }+ \text { False positives }+ \text { False negatives }+ \text { True negatives }} \times 100$, (4)Positive predictive value $=$
} 
$\frac{\text { True negatives }}{\text { True negatives }+ \text { False negatives }} \times 100$. The difference of sensitivity and specificity of $4 \mathrm{Mb}$ and $10 \mathrm{Mb}$ resolutions was determined by McNemar test. Statistical significance was defined when $\mathrm{P}<0.05$.

\section{Results}

\section{Trophectoderm Biopsy and Peripheral Blood Chromosome Analysis of Born Babies}

After research and inquiry, 17 families were recruited into the study. The results were described in the Table $1.58 .8 \%$ (10/17) of babies' analysis was consistent with $4 \mathrm{Mb}$ TE biopsies and 76.5\% (13/17) of babies' peripheral blood chromosome analysis was consistent with $10 \mathrm{Mb} \mathrm{TE}$ biopsies, which was not statistically significant $(\mathrm{P}=0.4646)$. $76.5 \%$ (13/17) of $\mathrm{TE}$ biopsies demonstrated congruent results between $4 \mathrm{Mb}$ and $10 \mathrm{Mb}$ resolution (Figure 1). Among them, 5.8\% (1/17) of TE biopsies were mosaic but the blood chromosomal analysis of the baby showed a segmental loss of neutral heterozygosity in Chromosome $\mathrm{X}$, which was different from the detection of TE biopsy and had unclear clinical significance (No.13). Interestingly, 11.8\% (2/17) of babies showed abnormal peripheral blood chromosome analysis with unclear clinical significance, which had normal NGS results in TE biopsies (No.1 and 16).

$23.5 \%$ (4/17) of TE biopsies were found chromosomally normal in $10 \mathrm{Mb}$ but abnormal in $4 \mathrm{Mb}$ resolution (Figure 1). 17.6\% (3/17) produced healthy babies with normal peripheral blood SNP-array. Another baby presented segmental haploid repeat in Chromosome $\mathrm{X}$ which had unclear clinical significance and were not consistent with 4Mb TE biopsy (No.8). For transparency, all karyotype profiles of both TE biopsies and blood samples analyzed in this study were shown in the Additional Figure 1.

\section{Sensitivity and specificity of $10 \mathrm{Mb}$ and $4 \mathrm{Mb}$ resolution}

For the purpose of assessing diagnostic accuracy of NGS in the $10 \mathrm{Mb}$ and $4 \mathrm{Mb}$ resolutions, sensitivity, specificity, PPV, NPV and diagnostic accuracy was calculated (Table 2). We analyzed them by classifying concordance of the TE and peripheral blood samples as true positive (abnormal TE, abnormal blood sample), true negative (normal TE, abnormal blood sample), false negative (normal TE, abnormal blood sample), and false positive (abnormal TE, normal blood sample). Based on this estimate, sensitivity (i.e., the probability of an abnormal blood sample being diagnosed from a TE biopsy) of $10 \mathrm{Mb}$ and $4 \mathrm{Mb}$ resolution was low, but specificity (i.e., the probability of a normal blood sample being diagnosed form a TE biopsy), PPV and diagnostic accuracy of $10 \mathrm{Mb}$ resolution were higher than $4 \mathrm{Mb}$. No statistical significance was found in sensitivity and specificity between $4 \mathrm{Mb}$ and $10 \mathrm{Mb}$ resolutions. Furthermore, the consistency between peripheral blood samples and TE biopsies were quite weak (Kappa value $\leq 0.4$ ).

\section{Discussion}

First of all, our results manifested that only $58.8 \%$ and $76.5 \%$ of babies had congruent peripheral chromosomal results with TE biopsies in $4 \mathrm{Mb}$ and $10 \mathrm{Mb}$ resolutions, respectively. $11.8 \%(2 / 17)$ of babies whose TE biopsies were euploid in both $4 \mathrm{Mb}$ and $10 \mathrm{Mb}$ resolutions showed abnormal peripheral blood chromosome analysis (No.1 and No. 16). 17.6\% (3/17) of aberrant embryos detected in the $4 \mathrm{Mb}$ resolution produced normal babies (No.5,6,7). Moreover, 2 babies' abnormal chromosomes were not similar with TE biopsies (No. 8 and 13). 
A number of previous studies adopted TE biopsies as a suitable source for PGT(10, 16, 17). However, the accuracy of TE biopsy for PGT to determine an euploid embryo is still a controversial issue. It is because TE biopsy is not a direct way to reflect ICM and just obtains 5-10 TE cells for subsequently diagnosing an embryo. For example, if certain abnormal chromosomal disjunction occurred in the TE cells, the TE biopsy might diagnose an euploid ICM as mosaicism or aneuploidy. It was reported that the concordance between TE and ICM was established in $62.1 \%$ of the embryos analyzed(8). And in Victor's research, there were 5 out of 100 aneuploidy embryos which had euploid ICM(7). In addition, the limited number of biopsied cells from a blastocyte that contains a few hundred TE cells makes the rate of mosaicism and aneuploidies vary widely $(3,18)$.

Another important reason for the inaccuracy of the TE biopsy is that chromosomal mosaicism, the presence of two or more distinct cell populations, and aneuploidies are prevalent throughout human pre- and post-implantation development $(19,20)$. Many factors conduce to the phenomenon, such as controlled ovarian hyperstimulation, embryo culture condition and so on(19). Investigated by Babariya and colleagues, they considered that during the first few mitotic divisions following fertilization, control of the cell cycle was more relaxed, permitting DNA double strand breaks to occur and persist through cell division(21). Those aberrations could be rescued after further embryo development by different pathways: anaphase-lag, nondisjunction, or chromosome demolition correction or clonal depletion correction $(19,22)$. Anaphase-lag correction describes that the process of anaphase lagging reverts the reciprocal chromosomes back to disomy. Nondisjunction correction describes that the three chromosomes will be divided into 2 daughter cells unevenly, one with 2 chromosomes and the other with 4 chromosomes. Then the tetrasomic cell will be depleted by apoptosis. Chromosome demolition correction indicates that aneuploid cells can give rise to two diploid cells by deliberate fragmentation of one of the three chromosomes during metaphase or anaphase. Clonal depletion correction depicts that aneuploid cells will be depleted by apoptosis or reduced proliferation. However, the potential of embryo to develop to a baby largely depends on the cell lineage and sufficient euploid cells. In Bolton et al.'s mouse model, they revealed that aneuploid cells in the fetal linage were eliminated by apoptosis, whereas those in the placental lineage showed severe proliferative defects(23). And they also showed that mosaic embryos have full developmental potential when they contained sufficient normal cells(23).

Laboratory error may also contribute to the inaccuracy. Assuming a normal euploid cell might have been biopsied, the laboratory assessment might be inaccurate and provide a misdiagnosis of aneuploidy(24). So, then some embryos with potential development are inadvertently being classified as unviable and lost chance to produce a healthy baby. There is no doubt that it is a big hit to poorprognosis patients who have only one embryo to transfer.

Despite the small patient numbers, the results we yielded here were consistent with another 3 aforementioned studies (2-4) in the regard that mosaic or aneuploid embryo could have potential to give birth to a healthy baby (No. 5,6,7 and 8). To the best of our knowledge, there is no report on chromosomal results of PGT babies after transferring euploid embryos hitherto. But if the accuracy of TE biopsy is doubtful, the chromosomal analysis of those babies produced by euploid PGT embryos is worthwhile to concern. Therefore, it is the first report that presumed euploid embryos could possibly 
produce babies with chromosomal abnormalities.

Although implanting mosaic or aneuploid embryos reduces the pregnancy rate(25), it could be a try for those patients with only an aneuploid embryo according to our investigation. Some researchers also commended that IVF clinicians could transfer abnormal embryos according the type of involved chromosomes and the degree of mosaicism of human embryo(3,18,19). Like, trisomies 2,3, 7 and 8 are the most frequently detected mosaic autosomal trisomies on chorionic villus sampling (CVS) and normally lead to a chromosomally normal fetus(26). Our data found segmental aneuploid chromosomes 2, 7, 16, 19, 20, X could produce healthy babies, especially for chromosomes 16 and 19, which had high contents of GC that would easily cause laboratory errors. Accordingly, we strongly agree with the recommendations published by Preimplantation Genetic Diagnosis International Society (PGDIS) that a prenatal testing is necessarily required after PGT, no matter whether the transferred embryo is normal or aberrant(27). The development of the fetus should be carefully observed, especially for embryos with mosaicism and aneuploidies during the conception. Once abnormal situation happened, it should take quick action to terminate pregnancy.

Additionally, we also calculated the sensitivity, specificity, PPV, NPV and accuracy of $10 \mathrm{Mb}$ and $4 \mathrm{Mb}$ resolutions, based on the chromosomal results of born babies as "gold standard". Although the sensitivity is low $(25 \%), 10 \mathrm{Mb}$ had higher specificity $(100 \%)$, PPV (100\%) and diagnostic accuracy (82.4\%) compared to the $4 \mathrm{Mb}$. As we all know, $4 \mathrm{Mb}$ have higher resolution and sensitivity than $10 \mathrm{Mb}$. However, such a high resolution screens out some abnormal embryos with subsequent developmental potential, making them discarded and lose chance to be implanted. Nonetheless, a relatively rough resolution $(10 \mathrm{Mb})$ assured the detection capability of confirming newborns with normal chromosomal profiles, that is, providing an opportunity to those slightly abnormal embryos for self-correction. In 2018 , a study pointed out the ability to detect a segmental aneuploidy of $<10 \mathrm{Mb}$ is not guaranteed and multiple other factors must be taken into account when using the technology for an inherited segmental change(28). The low Kappa values that reflected the predictive value for chromosomal profiles between TE biopsies and born baby confirmed the possible self-correction mechanisms during the development of embryos. But it is widely acknowleged that TE biopsy is less invasive and much safer for PGT. We should not deny its superiority in PGT and need to find a way to improve its application. In our experience, we suggest that we should analyze TE biopsy in both $10 \mathrm{Mb}$ and $4 \mathrm{Mb}$ resolutions to uncover severely adverse chromosomal aberrations but use $10 \mathrm{Mb}$ resolution to guide transfer.

Although our study firstly pointed out the inconsistency among TE biopsy of different NGS resolutions and peripheral blood samples of born babies, some inevitable limitations need to be acknowledged and discussed here. Firstly, it is a retrospective and observational study with a quite small sample size. Secondly, we just compared TE biopsy in $10 \mathrm{Mb}$ and $4 \mathrm{Mb}$ resolutions. More precise or rougher resolution is supposed to be investigated in the further studies. Finally, it is worth noting that NGS and SNP array in our investigation are two distinct techniques using different platforms, which might result in analysis bias. For the data we and other teams provided(2-4), a research with large sample number is required in the future.

\section{Conclusions}

Our data demonstrated that the chromosomal results between peripheral blood samples and TE biopsies 
of born babies were not completely congruent. Aneuploid and mosaic embryos had potential to produce healthy babies, whereas normal embryos also had chance to produce babies with chromosomal abnormality. In spite of low sensitivity of both resolutions, $10 \mathrm{Mb}$ had higher specificity, positive predictive value and diagnostic accuracy than $4 \mathrm{Mb}$. We also suggested that TE biopsy be analyzed in both $10 \mathrm{Mb}$ and $4 \mathrm{Mb}$ resolutions to uncover severely adverse chromosomal aberrations but use $10 \mathrm{Mb}$ resolution to guide transfer.

\section{List of abbreviations}

PGT: preimplantation genetic testing; ICM: inner cell mass; CNVs: copy number variations; FISH: fluorescence in situ hybridization; aCGH: array comparative genomic hybridization; SNP: single nucleotide polymorphism; NGS: next generation sequencing; ICSI: intracytoplasmic sperm injection; FET: frozen embryo transfer; HRT: hormone replacement therapy; NC: natural cycle; TE: trophectoderm; PPV: positive predictive value; NPV: negative predictive value; CVS: chorionic villus sampling.

\section{Declarations}

Ethics approval and consent to participate: The study was approved by the Institutional Review Board, Department of Reproductive Center, Xiangya Hospital, Central South University (No.202006). All informed consent forms from participants were signed and received.

Consent for publication: Not Applicable.

Availability of data and material: The datasets generated during and/or analyzed during the current study are available from the corresponding author on reasonable request.

Competing interests: Authors declare that they have no conflict of interest.

Funding: The study was supported by Technology Innovation Guidance Program of Hunan Province (2017SK50103).

Authors' contribution: All authors contributed to the study conception and design. Material preparation, data collection and analysis were performed by Zhongyuan Yao, Xiaoxia Wang, Jun Zeng, Jing Zhao, Qiuping Xia, Lei Zhang, Lingqian Wu and Yanping Li. The first draft of the manuscript was written by Zhongyuan Yao and Xiaoxia Wang. All authors commented on previous versions of the manuscript. All authors read and approved the final manuscript.

Acknowledgements: The authors thank Dr. Ge Yang and M.Sc Xinlei Zhu for their helps in statistical analysis. The authors also thank all staff's hard work in Department of Reproductive Medicine, Xiangya Hospital, Central South University, without whom the study could not be carried out.

\section{Footnotes:}

Zhongyuan Yao and Xiaoxia Wang contribute equally in the study.

\section{References:}

1. Handyside AH, Pattinson JK, Penketh RJ, Delhanty JD, Winston RM, Tuddenham EG. Biopsy of human preimplantation embryos and sexing by DNA amplification. Lancet 1989;1:347-9. doi:10.1016/s0140-6736(89)91723-6

2. Greco E, Minasi MG, Fiorentino F. Healthy Babies after Intrauterine Transfer of Mosaic Aneuploid Blastocysts. N Engl J Med 2015;373:2089-90. doi:10.1056/NEJMc1500421

3. Gleicher N, Vidali A, Braverman J, Kushnir VA, Barad DH, Hudson C, Wu Y, Wang Q, Zhang L, Albertini DF. Accuracy of preimplantation genetic screening (PGS) is compromised by degree of 
mosaicism of human embryos. Reprod Biol Endocrin 2016;14. doi:10.1186/s12958-016-0193-6

4. Kahraman S, Cetinkaya M, Yuksel B, Yesil M, Pirkevi Cetinkaya C. The birth of a baby with mosaicism resulting from a known mosaic embryo transfer: a case report. Hum Reprod 2020;35:727-33. doi:10.1093/humrep/dez309

5. Vera-Rodriguez M, Diez-Juan A, Jimenez-Almazan J, Martinez S, Navarro R, Peinado V, Mercader A, Meseguer M, Blesa D, Moreno I et al.. Origin and composition of cell-free DNA in spent medium from human embryo culture during preimplantation development. Hum Reprod 2018;33:745-56. doi:10.1093/humrep/dey028

6. Treff NR, Fedick A, Tao X, Devkota B, Taylor D, Scott RT. Evaluation of targeted next-generation sequencing - based preimplantation genetic diagnosis of monogenic disease. Fertil Steril 2013;99:137784. doi:10.1016/j.fertnstert.2012.12.018

7. Victor AR, Griffin DK, Brake AJ, Tyndall JC, Murphy AE, Lepkowsky LT, Lal A, Zouves CG, Barnes FL, McCoy RC et al.. Assessment of aneuploidy concordance between clinical trophectoderm biopsy and blastocyst. Hum Reprod 2019;34:181-92. doi:10.1093/humrep/dey327

8. Popovic M, Dheedene A, Christodoulou C, Taelman J, Dhaenens L, Van Nieuwerburgh F, Deforce D, Van den Abbeel E, De Sutter P, Menten B et al.. Chromosomal mosaicism in human blastocysts: the ultimate challenge of preimplantation genetic testing? Hum Reprod 2018;33:1342-54. doi:10.1093/humrep/dey106

9. Maxwell SM, Colls P, Hodes-Wertz B, McCulloh DH, McCaffrey C, Wells D, Munné S, Grifo JA. Why do euploid embryos miscarry? A case-control study comparing the rate of aneuploidy within presumed euploid embryos that resulted in miscarriage or live birth using next-generation sequencing. Fertil Steril 2016;106:1414-9. doi:10.1016/j.fertnstert.2016.08.017

10. Liu X, Fan Q, Wang J, Li R, Xu Y, Guo J, Wang Y, Zeng Y, Ding C, Cai B et al.. Higher chromosomal abnormality rate in blastocysts from young patients with idiopathic recurrent pregnancy loss. Fertil Steril 2020;113:853-64. doi:10.1016/j.fertnstert.2019.11.016

11. Gardner DK, Schoolcraft WB. In vitro culture of human blastocyst. Towards Reproductive Certainty Infertility \& Genetics Beyond 1999.

12. Zhao J, Yan Y, Huang X, Sun L, Li Y. Blastocoele expansion: an important parameter for predicting clinical success pregnancy after frozen-warmed blastocysts transfer. Reprod Biol Endocrinol 2019;17:15. doi:10.1186/s12958-019-0454-2

13. Jiao J, Shi B, Sagnelli M, Yang D, Yao Y, Li W, Shao L, Lu S, Li D, Wang X. Minimally invasive preimplantation genetic testing using blastocyst culture medium. Hum Reprod 2019;34:1369-79. doi:10.1093/humrep/dez075

14. Zong C, Lu S, Chapman AR, Xie XS. Genome-wide detection of single-nucleotide and copynumber variations of a single human cell. Science 2012;338:1622-6. doi:10.1126/science.1229164

15. Qi Q, Lu S, Zhou X, Yao F, Hao N, Yin G, Li W, Bai J, Li N, Cram DS. Copy number variation sequencing-based prenatal diagnosis using cell-free fetal DNA in amniotic fluid. Prenat Diagn 2016;36:576-83. doi:10.1002/pd.4830

16. Zhao H, Tao W, Li M, Liu H, Wu K, Ma S. Comparison of two protocols of blastocyst biopsy submitted to preimplantation genetic testing for aneuploidies: a randomized controlled trial. Arch Gynecol Obstet 2019;299:1487-93. doi:10.1007/s00404-019-05084-1

17. Munné S, Kaplan B, Frattarelli JL, Child T, Nakhuda G, Shamma FN, Silverberg K, Kalista T, Handyside AH, Katz-Jaffe M et al.. Preimplantation genetic testing for aneuploidy versus morphology as selection criteria for single frozen-thawed embryo transfer in good-prognosis patients: a multicenter 
randomized clinical trial. Fertil Steril 2019;112:1071-9. doi:10.1016/j.fertnstert.2019.07.1346

18. Maxwell SM, Colls P, Hodes-Wertz B, McCulloh DH, McCaffrey C, Wells D, Munné S, Grifo JA. Why do euploid embryos miscarry? A case-control study comparing the rate of aneuploidy within presumed euploid embryos that resulted in miscarriage or live birth using next-generation sequencing. Fertil Steril 2016;106:1414-9. doi:10.1016/j.fertnstert.2016.08.017

19. Taylor TH, Gitlin SA, Patrick JL, Crain JL, Wilson JM, Griffin DK. The origin, mechanisms, incidence and clinical consequences of chromosomal mosaicism in humans. Hum Reprod Update 2014;20:571-81. doi:10.1093/humupd/dmu016

20. Vanneste E, Voet T, Le Caignec C, Ampe M, Konings P, Melotte C, Debrock S, Amyere M, Vikkula M, Schuit F et al.. Chromosome instability is common in human cleavage-stage embryos. Nat Med 2009;15:577-83. doi:10.1038/nm.1924

21. Babariya D, Fragouli E, Alfarawati S, Spath K, Wells D. The incidence and origin of segmental aneuploidy in human oocytes and preimplantation embryos. Hum Reprod 2017;32:2549-60. doi:10.1093/humrep/dex324

22. Munné S, Velilla E, Colls P, Bermudez MG, Vemuri MC, Steuerwald N, Garrisi J, Cohen J. Selfcorrection of chromosomally abnormal embryos in culture and implications for stem cell production. Fertil Steril 2005;84:1328-34. doi:10.1016/j.fertnstert.2005.06.025

23. Bolton H, Graham SJL, Van der Aa N, Kumar P, Theunis K, Fernandez Gallardo E, Voet T, Zernicka-Goetz M. Mouse model of chromosome mosaicism reveals lineage-specific depletion of aneuploid cells and normal developmental potential. Nat Commun 2016;7. doi:10.1038/ncomms11165

24. Scott RT, Ferry K, Su J, Tao X, Scott K, Treff NR. Comprehensive chromosome screening is highly predictive of the reproductive potential of human embryos: a prospective, blinded, nonselection study. Fertil Steril 2012;97:870-5. doi:10.1016/j.fertnstert.2012.01.104

25. Fragouli E, Alfarawati S, Spath K, Babariya D, Tarozzi N, Borini A, Wells D. Analysis of implantation and ongoing pregnancy rates following the transfer of mosaic diploid-aneuploid blastocysts. Hum Genet 2017;136:805-19. doi:10.1007/s00439-017-1797-4

26. Malvestiti F, Agrati C, Grimi B, Pompilii E, Izzi C, Martinoni L, Gaetani E, Liuti MR, Trotta A, Maggi F et al.. Interpreting mosaicism in chorionic villi: results of a monocentric series of 1001 mosaics in chorionic villi with follow-up amniocentesis. Prenatal Diag 2015;35:1117-27. doi:10.1002/pd.4656

27. Redaktsiia. PGDIS Position Statement on the Transfer of Mosaic Embryos in Preimpatation Genetic Testing for Aneuploidy (PGT-A). Problemy reproduktsii 2019;25:8. doi:10.17116/repro2019250418

28. Cuman C, Beyer CE, Brodie D, Fullston T, Lin JI, Willats E, Zander-Fox D, Mullen J. Defining the limits of detection for chromosome rearrangements in the preimplantation embryo using next generation sequencing. Hum Reprod 2018;33:1566-76. doi:10.1093/humrep/dey227

\section{Figure legends:}

Figure 1. Pie chart of TE-peripherical blood in two different NGS resolutions. Abbreviations: TE, trophectoderm; NGS, next generation Sequencing.

Additional Figure 1. Karyotype profiles of 17 TE biopsies and peripheral blood samples. For TE biopsies, NGS-based PGT technique was used. For peripheral blood samples, SNP array was applied and only chromosomally abnormal profiles were provided. 
Table 1. The chromosomal analysis of trophectoderm biopsies and peripheral blood samples of born babies

\begin{tabular}{|c|c|c|c|c|c|}
\hline $\begin{array}{l}\text { Sampl } \\
\text { e ID }\end{array}$ & $\begin{array}{l}\text { Gardner } \\
\text { Grade }\end{array}$ & Reason of PGT & $\begin{array}{l}\text { CNV result of trophectoderm biopsies } \\
\qquad(4 \mathrm{Mb})\end{array}$ & $\begin{array}{l}\text { CNV result of trophectoderm biopsies } \\
\qquad(10 \mathrm{Mp})\end{array}$ & CNV result of born babies \\
\hline \multicolumn{6}{|c|}{ Concordance between $4 \mathrm{Mb}$ and $10 \mathrm{Mb}$ sequencing depth } \\
\hline \multirow[t]{3}{*}{1} & & & $46, \mathrm{XN}$ & $46, \mathrm{XN}$ & $\operatorname{arr} 22 q 11.21(18,510,240-$ \\
\hline & $3 \mathrm{BB}$ & $46, X X, t(7 ; 9)(q 22 ; q 22)$ & & & $19,028,462) \times 3$ \\
\hline & & & & & Haploid repeat \\
\hline 2 & 4BB & $46, \mathrm{XX}, 1 \mathrm{qh}+$ & $46, \mathrm{XN}$ & $46, \mathrm{XN}$ & $46, \mathrm{XN}$ \\
\hline 3 & 4BB & $45, X Y, \operatorname{der}(13 ; 14)(q 10 ; q 10)$ & $46, \mathrm{XN}$ & $46, \mathrm{XN}$ & $46, \mathrm{XN}$ \\
\hline 4 & $5 \mathrm{CB}$ & Abnormal Gestation & $46, \mathrm{XN}$ & $46, \mathrm{XN}$ & $46, \mathrm{XN}$ \\
\hline 9 & 4BB & Repeated Implantation Failure & $46, \mathrm{XN}$ & $46, \mathrm{XN}$ & $46, \mathrm{XN}$ \\
\hline 10 & $4 \mathrm{BC}$ & 46,XY,t(10;11)(q11;p12) & $46, \mathrm{XN}$ & $46, \mathrm{XN}$ & $46, \mathrm{XN}$ \\
\hline 11 & 5BB & Repeated Implantation Failure & $46, \mathrm{XN}$ & $46, \mathrm{XN}$ & $46, \mathrm{XN}$ \\
\hline 12 & $4 \mathrm{BB}$ & $45, X[8] / 46, X X[197]$ & $46, \mathrm{XN}$ & $46, \mathrm{XN}$ & $46, \mathrm{XN}$ \\
\hline \multirow[t]{3}{*}{13} & & & $46, \mathrm{XN},+2 \mathrm{q}(\mathrm{q} 11.2 \rightarrow \mathrm{q} 22.1, \sim 44 \mathrm{M}, \times 3, \mathrm{mo}$ & $46, \mathrm{XN},+2 \mathrm{q}(\mathrm{q} 11.2 \rightarrow \mathrm{q} 22.1, \sim 44 \mathrm{M}, \times 3, \mathrm{mo}$ & $\operatorname{arrXq13.3q21.1(74,630,390-}$ \\
\hline & $5 \mathrm{BC}$ & 45,XY,rob(13;14)(q10;q10) & $\mathrm{s}, \sim 40 \%)$ & $\mathrm{s}, \sim 40 \%)$ & $80,675,155) \times 2$ \\
\hline & & & & & loss of neutral heterozygosity \\
\hline 14 & $5 \mathrm{BB}$ & Recurrent Miscarriage & $46, \mathrm{XN}$ & $46, \mathrm{XN}$ & $46, \mathrm{XN}$ \\
\hline 15 & $5 \mathrm{CB}$ & $46, X X, t(6 ; 7)(q 15 ; q 22)$ & $46, \mathrm{XN}$ & $46, \mathrm{XN}$ & $46, \mathrm{XN}$ \\
\hline \multirow[t]{3}{*}{16} & & & $46, \mathrm{XN}$ & $46, \mathrm{XN}$ & $\operatorname{arr1q25.1q31.1(175919492-~}$ \\
\hline & $4 \mathrm{BB}$ & $46, X Y, t(3 ; 10)(q 25 ; q 22)$ & & & $190108881) \times 2$ \\
\hline & & & & & loss of neutral heterozygosity \\
\hline 17 & $6 \mathrm{BB}$ & $46, X Y, \operatorname{inv}(8)(\mathrm{p} 23.1 ; \mathrm{q} 11.23)$ & $46, \mathrm{XN}$ & $46, \mathrm{XN}$ & $46, \mathrm{XN}$ \\
\hline \multicolumn{6}{|c|}{ Discordance between $4 \mathrm{Mb}$ and $10 \mathrm{Mb}$ sequencing depth } \\
\hline 5 & $4 \mathrm{BB}$ & $45, \mathrm{XX}, \operatorname{der}(13 ; 14)(\mathrm{q} 10 ; \mathrm{q} 10)$ & $\begin{array}{l}46, X X,+X q(q 11.1 \rightarrow q 12, \sim 5.0 \mathrm{Mb}, \times 3),+ \\
X q(q 13.2 \rightarrow q 21.1, \sim 5.9 \mathrm{Mb}, \times 3),+16 \mathrm{p}(\mathrm{p} 1\end{array}$ & $46, \mathrm{XN}$ & $46, \mathrm{XN}$ \\
\hline
\end{tabular}


6

$\begin{array}{lll} & 5 B C & 46, X X, t(5 ; 10)(\mathrm{q} 15 ; \mathrm{p} 11.2) \\ 7 & 3 \mathrm{CB} & 45 \mathrm{XX}, 1 \mathrm{qhder}(13,14)(\mathrm{q} 10, \mathrm{q} 10) \\ 8 & & \\ & 3 \mathrm{BB} & \text { Recurrent Miscarriage }\end{array}$

$46, X X,+X q(q 11.1 \rightarrow q 12, \sim 5 M b, \times 3),+X$

$\mathrm{q}(\mathrm{q} 13.2 \rightarrow \mathrm{q} 21.1, \sim 11 \mathrm{Mb}, \times 3),+7 \mathrm{q}(\mathrm{q} 11.2$

$1 \rightarrow \mathrm{q} 11.21, \sim 4 \mathrm{Mb}, \times 3),+16 \mathrm{p}(\mathrm{p} 11.2 \rightarrow \mathrm{p} 11$

$.2, \sim 4 \mathrm{Mb}, \times 3),+19 \mathrm{p}(\mathrm{p} 12 \rightarrow \mathrm{p} 11, \sim 4.3 \mathrm{Mb}$,

$\times 4)$

$46, \mathrm{XN},+16 \mathrm{q}(\mathrm{q} 21 \rightarrow \mathrm{q} 21, \sim 4.1 \mathrm{Mb}, \times 3)$

$46, X N,+20 \mathrm{q}(\mathrm{q} 11.21 \rightarrow \mathrm{q} 11.23, \sim 5.9 \mathrm{Mb}$,

$\times 3)$
$46, \mathrm{XN}$

$46, \mathrm{XN}$

$46, \mathrm{XN}$

$\operatorname{arrXp22.31(6,456,940-~}$

$8,135,053) \times 2$

Haploid repeat

Table 2 Sensitivity, specificity, PPV, NPV and accuracy for TE biopsy at the resolution of 4Mb and 10Mb NGS.

\begin{tabular}{llllllll}
\hline & $\begin{array}{l}\text { Sensitivity } \\
(\%)\end{array}$ & $\begin{array}{l}\text { Specificity } \\
(\%)\end{array}$ & $\begin{array}{l}\text { PPV } \\
(\%)\end{array}$ & $\begin{array}{l}\text { NPV } \\
(\%)\end{array}$ & $\begin{array}{l}\text { Accuracy } \\
(\%)\end{array}$ & Kappa value & P value \\
\hline $4 \mathrm{Mb}$ & 50 & 76.9 & 40 & 83.3 & 70.6 & 0.248 & $>0.05$ \\
$10 \mathrm{Mb}$ & 25 & 100 & 100 & 81.3 & 82.4 & 0.338 & \\
\hline
\end{tabular}

Abbreviations: PPV, positive predictive value; NPV, negative predictive value; TE, trophectoderm; NGS, next generation sequencing. 


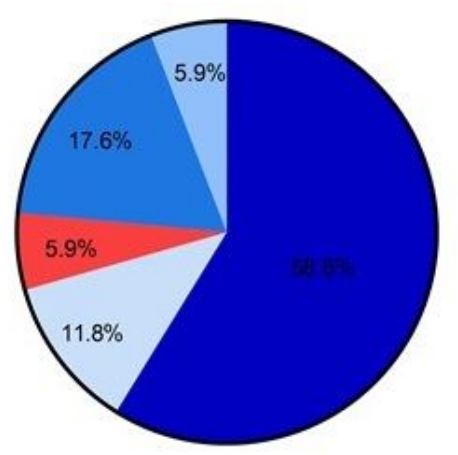

Normal $10 \mathrm{Mb} \mathrm{TE}$, normal 4Mb TE, chromosomally normal baby Normal $10 \mathrm{Mb} \mathrm{TE}$, normal $4 \mathrm{Mb} \mathrm{TE}$, chromosomally abnormal baby

- Abnormal $10 \mathrm{Mb} \mathrm{TE}$, abnormal $4 \mathrm{Mb} \mathrm{TE}$, chromosomally abnormal baby

- Normal $10 \mathrm{Mb} \mathrm{TE}$, abnormal $4 \mathrm{Mb} \mathrm{TE}$, chromosomally normal baby

= Normal 10Mb TE, abnormal 4Mb TE, chromosomally abnormal baby

Total $=17$

\section{Figure 1}

Pie chart of TE-peripherical blood in two different NGS resolutions. Abbreviations: TE, trophectoderm; NGS, next generation Sequencing.

\section{Supplementary Files}

This is a list of supplementary files associated with this preprint. Click to download.

- AdditionalFigure1.pdf 